\title{
INTEGRATED AND OBJECTIVE-ORIENTED STATISTICAL PROCESS CONTROL
}

\author{
ELART VON COLLANI \\ Institute of Economy \\ University of Würzburg \\ D-97070 Würzburg \\ Germany
}

\begin{abstract}
Global competition and increased environmental concern have emphasized the significance of process quality for an economic success. Statistical Process Control (SPC) is considered as one of the major tools for better quality. However, there are many opinions about the nature, the role and the results of SPC in industry. If SPC is understood and implemented correctly, then it should not only lead to "a reduction of waste, shorter throughput times and greater reliability of supply, but also in a better understanding of the processes and their variation" as Does, Roes and Trip formulated it in [15]. SPC should ultimately lead to "an operational management of the continuous improvement of processes." The aim of an industrial production process is to make profit, thus quality should by measured by process profitability. From a more operational point of view highest quality, i.e. highest profitability, is reached by producing with high conformance to an appropriately selected target value, and quality improvement means further reduction of variation around the target value. Thus, understanding the inherent variation represents the key for controlling "profitability" and for being able to implement a system of continuous "quality improvement". The inherent variations reflect the complex relations between everything concerning the production process and results in uncertainty about the future development. Exactly at this point stochastics, the science of uncertainty, enters the stage. For controlling profitability the relations and the resulting variations have to be taken appropriately into account by an integrated and objective-oriented stochastic approach.
\end{abstract}

\section{INTRODUCTION}

Most, if not every problem is the result of or is related to uncertainty. Stochastics, the science of uncertainty with probability theory as its theoretical part and statistics as its empirical part offers the necessary means for solving almost any problem as good as possible in a quantitative and reliable way. Moreover, having identified uncertainty 
as source of a problem makes stochastics the scientific key for continuous improvement of knowledge and methods by means of the cycle observation, evaluation, modeling, application which is represented graphically below.

The scientific cycle means that everything starts with observing a real phenomenon by experiments. The next step is to evaluate the observed results including the inherent random variation by scientific procedures. The evaluation leads to a quantitative model describing the variations exhibited by the phenomenon. Once the model is available, it can be utilized for application. Any application leads to improved knowledge and further observations, which again are subject of evaluation, leading to an improvements of the model, etc.

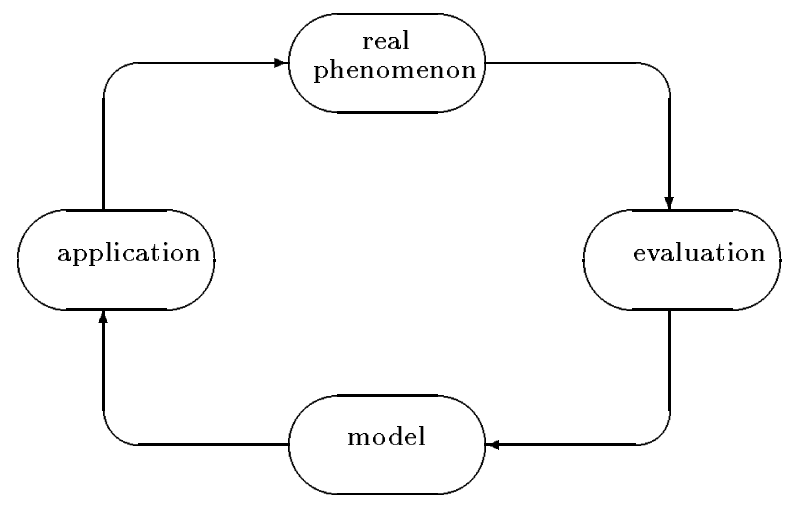

Figure 1: Scientific Cycle.

In order to make the scientific cycle rotate field specialists and stochasticians must cooperate. In a first step, the stochastician develops a stochastic model for the inherent variations based on the available knowledge made available by the field specialist. The second step consists in deriving the "statistical" procedures to be applied in order to answer questions and to solve problems. Generally, neither model nor procedures can be taken from standards, but have to be developed by a custom made approach implying that the stochastician has to take over the leadership in an inter-disciplinary team. Does et al [15] state "We will even go so far as to state that the more the statistician succeeds in attaining the role of supervisor and intitiator instead of calculator, the better he is able to carry out his job."

The arising problems can be classified in

- measurement problems for determining the actual value of a quantity of interest,

- prediction problems for forecasting a future event, and 
- decision problems, where different alternatives have to be compared or excluded.

Such problems and the necessity to solve them occur in any branch of science and in any part of society explaining the universal use of stochastics models and statistical methods.

Figure 2 displays a diagram of the cyclic steps - called Product Cycle-leading to improved or entirely new products. Each step involves many problems and decisions and is linked to the other steps and all of them to the center represented by the process management and product marketing. Any solution for the arising problems not taking appropriately into account the inherent variability caused by technical, environmental, social and other features must be judged hazardous and will lead generally to an inferior result or even to failure. The characteristic variability of real-world phenomena is the outcome of existing relations which connect everything with everything over space and time implying

- that there are no pure cause-effect relations,

- that generally future developments cannot be predicted with certainty, and

- that deterministic modeling of real-world phenomena is generally inappropriate.

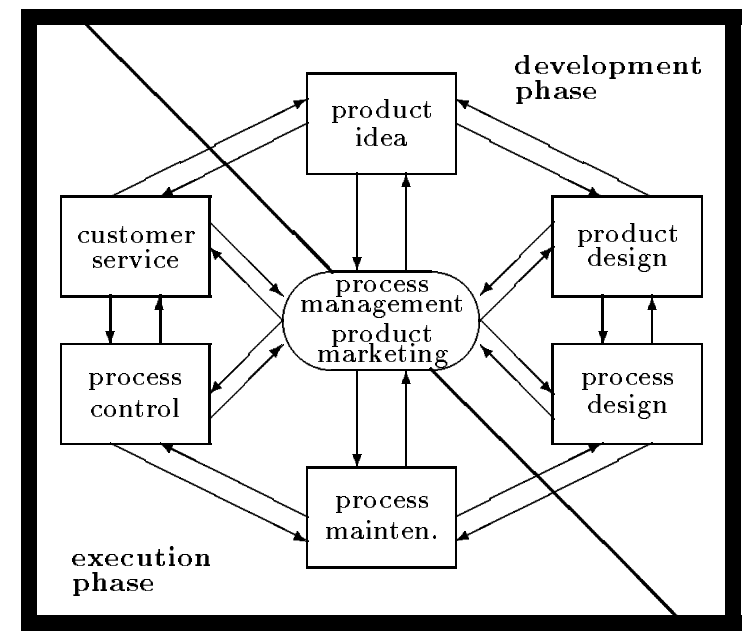

Figure 2: The Product Cycle.

As shown in Figure 2 the Product Cycle can be divided into a development phase and an execution phase. Each of them poses specific problems and each of them is related to the others. In order to arrive at appropriate solutions for any one of the existing problems, the following points have to be considered necessarily:

1. The ultimate aim of production. 
2. The inherent uncertainty.

3. The procedures to be implemented.

4. The relations between the problems and their solutions.

\section{ULTIMATE AIM}

From the producer's viewpoint the ultimate aim of producing and selling a product is to make profit. Therefore, for a producer quality of a production process is exclusively determined by process profitability. From the consumer's viewpoint, however, product quality is of major interest. However, the criteria for evaluating product quality might be different for different consumers. Moreover, product quality from the viewpoint of public or social communities might again be entirely different illustrating the fact that quality is not an absolute quantity but a relative one depending on the underlying objective.

Profitability depends on the solutions of many problems, and for evaluating the quality of each single solution, it has to be evaluated by its contribution to profitability. Thus, profitability serves as comparison criterion with respect to quality for the product, for the production process, for marketing, for customer service and even for the product idea. In order to use profitability as a quality comparison criterion it must necessarily be defined quantitatively depending on the entirety of the solutions.

For defining profitability two cases have to be distinguished.

- Profitability in the long run.

- Profitability in the finite run.

If the number of items to be produced is not known beforehand, but the aim is to produce and sell as many products as possible over an unknown time, then the first case is appropriate. The second case is appropriate if the process is installed for producing a fixed and small number number of items. Since the second scenario refers to rather special situations, this paper is restricted to the first case.

It is assumed that the output of the production process can be divided into suitable items, which exhibit a certain variability in their performance characteristics. Let $X_{i}$ denote the performance characteristics of the $i$ th produced item and let $N(t)$ denote the number of items produced up to the time $t$ and $G(t)$ denote the profit derived from these items. Then the profit per item in the long run denoted by $\Pi$ is defined by the limit:

$$
\Pi=\lim _{t \rightarrow \infty} \frac{G(t)}{N(t)}
$$


Once (1) has been derived for a process one can compare quantitatively different designs for the development phase and different strategies for the execution phase in order to determine those which increase profitability.

\subsection{Uncertainty and Procedures}

The derivation of (1) must necessarily take into account the inherent uncertainty about the development of any of the involved quantities. For this end the variability of the phenomena in question has to be modeled by suitably selected probability distribution. The more relevant information about a phenomenon are available, the better the probability distribution can be chosen for describing the variability.

A first but very important step in the modeling task involves the decision about relevant information. The questions to be answered refer to the relevant information and how to make it available. An evaluation of the state of information and a program for continuously improving it are two eminent important points.

Before the production process is started, the

- level of performance, and

- the way how to maintaining this level during the entire time of process operation

should be decided based on the available information. The first problem, although of great significance for an economic success, is not treated here. In a series of papers Kiesmüller has investigated related problems (compare Kiesmüller [22], [23], [24] and [25]).

Of course, both tasks are again closely related. If the performance level is fixed at a too high level, the cost of maintenance will be excessively high, thus yielding a low profitability. On the other hand, if the cost of maintenance is limited to a rather low level then the performance level will decrease resulting again in a low process profitability. Therefore, the appropriate performance level and the maintenance strategy can be decided upon in a rational way only by means of (1).

The performance level for an item $\mathrm{X}$ produced is defined by a probability distribution $F_{X}^{(\theta)}(x)$ of the relevant quality characteristics $X$, where $\theta$ is a possibly multi-dimensional distribution parameter. The process performance level is maintained over time, if for any process segment $\left(X_{1}, \ldots, X_{N}\right)$ and any $N \in \mathbb{N}$ the following two conditions with respect to the variations are met:

$$
F_{X_{1}, \ldots, X_{N}}^{\left(\theta_{1}, \ldots, \theta_{N}\right)}\left(x_{1}, \ldots, x_{N}\right)=\prod_{i=1}^{N} F_{X_{i}}^{\left(\theta_{i}\right)}\left(x_{i}\right)
$$




$$
F_{X_{i}}^{\left(\theta_{i}\right)}\left(x_{i}\right)=F_{X}^{(\theta)}\left(x_{i}\right) \quad \text { for any } i \in\{1, \ldots, N\}
$$

Of course, meeting strictly conditions (2) and (3) will not be possible, but the maintenance strategy should be designed in a way that the two conditions are almost met.

From the viewpoint of process maintenance and process control four aspects are of special interest.

\section{- External effects:}

1. 1. Process input.

2. 2. Environmental process conditions.

- Internal Effects:

1. 3. Process wear.

2. 4. Process disturbance.

Variations in the process input may affect the technical performance and lead to changes in the probability distribution of the performance characteristics. There are two ways to overcome the problems of varying input. The most common way is to monitor and sort the input by means of acceptance sampling plans. Another way would be to monitor the input process and react on any variation by a change in the process itself thus compensating the change of the input.

Generally, the technical performance of a production process depends on environmental conditions like temperature, pressure, cleanliness. If there are too large changes in the relevant conditions, the process performance will suffer. Generally the environmental conditions are continuously monitored and controlled by automatic control devices, for details compare for example Box \& Luceño [4].

Process wear and the related process aging may increase the proneness for process disturbances particularly for process failures or may directly worsen process performance. Wear and aging are compensated by means of continuous maintenance policies which remove wear and aging to a large extent by preventive process corrections before they can affect process profitability. Any correction action is called preventive, if it is performed before the state of the corresponding process part affects the joint probability distribution (2) markedly. The times of preventive corrections are fixed by means of process characteristics like operation time, or accumulated costs, or others which reflect the actual state of (2) or (5) and thus, announce an approaching considerable process deterioration. This leads to a characteristic feature of phenomena like process wear and aging. Their effects 
are to some extent predictable since their development is a known function of other, observable characteristics.

Finally, there are random process disturbances which occur like accidents. Unlike disturbances due to wear and aging there are no observable features which announce an approaching random disturbance. Their occurrence is independent of process characteristics like operation time, cost or number of produced items. The process proneness to disturbances is modeled by the disturbance intensity function, which sometimes is called hazard function of a lifetime distribution denoted by:

At time of production $t_{1}$ the intensity function $\lambda(t)$ completely determines the distribution of the time $\tau_{t_{1}}$ until the next occurring disturbance. The distribution function of $\tau_{t_{1}}$ is given by:

$$
G_{\tau_{t_{1}}}(y)=1-e^{-\int_{t_{1}}^{y} \lambda(t) d t}
$$

Of course, one aim of process design is to reduce the frequency of random process disturbances. However, experience shows that there is no production process without occasionally occurring disturbances. Generally, process disturbances cause a sudden and substantial change in the joint distribution (2) leading to a considerable decrease of process profitability. As these disturbances cannot be prevented, they must be detected early enough by process monitoring in order to limit the loss of profit. Process monitoring may be performed by observing characteristics which reflect the actual form of (2), for instance the process output $\left\{X_{i}\right\}$.

\subsection{Relations}

The different steps of a product-cycle are related to and affect each other. However, generally it would be too difficult if not impossible to solve the existing problems simultaneously. One way out of this difficulty is to answer the questions iteratively, where the solution on step $k$ takes into account the preceding solutions on step 1 to step $k-1$. For example, although maintenance and process control are closely connected, the strategy to select independently from process control in a first step an appropriate maintenance policy and in a second step, taking into account the results of the maintenance policy, an appropriate policy for process control yields an almost optimal result as shown in Frahm $[19]$.

Taking into account the relations assumes that the impact of e.g. product design, process 
design, warranty policy, etc. on the variation exhibited during the various process stages and model them by a joint probability distribution of all relevant features. One prerequisite for arriving at appropriate solutions is to adopt statistical thinking which has been advocated for many decades by the late Edward W. Deming. Statistical thinking means to recognize that random variations generated by stochastic relations cause the problems and any improvement must be based on a thorough investigation and stochastic modeling of the relevant relations.

The main topic of this paper is statistical process control. Therefore, it is assumed that the other steps of the production cycle have been successfully accomplished and hence only the results of these steps have to be considered, because process maintenance has an immediate impact on statistical process control, the former is briefly treated in the next section.

\section{PROCESS MAINTENANCE}

As mentioned earlier, process wear has different effects which have to be modeled differently. The gradually developing dependency of the output $X_{t}$ and its gradually developing deviation from target is modeled by $\theta(t)$. The gradually increasing disturbance intensity is modeled by $\lambda(t)$.

The two standard methods of process maintenance are on the one hand automatic process controllers (see for instance Box \& Luceño [4] and Dumitrescu [17], [16]), which continuously monitor the relevant process parameters and automatically adjust them, and on the other hand preventive replacements/renewals (see for instance Beichelt [2], [3]). Both types of procedures aim at keeping $\theta(t)$ and $\lambda(t)$ for all $t$ close to their respective targets $\theta_{0}$ and $\lambda_{0}$. Therefore, assuming that an appropriate process maintenance has been implemented implies:

$$
\begin{aligned}
& \theta(t) \approx \theta_{0} \text { for any } t>0 \\
& \lambda(t) \approx \lambda_{0} \text { for any } t>0
\end{aligned}
$$

From (7) it immediately follows that the disturbance intensity can be looked upon as constant implying that the exponential distribution with parameter $\lambda_{0}$ is an appropriate model for the random variable $\tau$. Assuming a different distribution of $\tau$ as for example in Rahim [28] and Rahim \& Banerjee [29] means that the maintenance policy does not remove wear and aging sufficiently well.

Of course, (6) is only guaranteed as long as there is no random disturbance. Therefore, 
it remains to solve the problem of detecting and removing the effects of random disturbances, which in general cannot be compensated by automatic process controllers or preventive corrections. Not to remove disturbances and to wait until the next preventive maintenance action could last too long and therefore may cause a considerable

\section{STATISTICAL PROCESS CONTROL}

The problem with random disturbances is to detect their occurrence. Once they are detected, it is assumed that they can be removed completely. From (7) and (6) it follows that removing a process disturbance can be looked upon as a process renewal.

For detecting a disturbance a process monitoring policy has to be implemented, i.e. a directive fixing the actions which have to be performed aiming at detecting any occurred disturbance. By its implementation the monitoring policy becomes a part of the process. In order to compare different monitoring policies we need an indicator reflecting their contributions to overall process profitability.

Any monitoring policy in the framework of statistical process control for detecting the occurrence of random disturbances is given by $\left\{\left(t_{i}, n_{i}, \gamma_{i}\right)\right\}_{i \in N}$, where $t_{i}$ is the $i$ th monitoring timepoint, $n_{i}$ is the sample size at time $t_{i}$, and $\gamma_{i}$ is the decision rule. For a more detailed presentation see v. Collani [5]. Here, we restrict ourselves to periodic monitoring policies, i.e.:

$$
t_{i+1}-t_{i}=h \quad \text { for } \quad i=1,2, \cdots \text { with } \quad t_{0}=0
$$

with fixed sample size $n_{i}=n$. Aim of sampling at time $t_{i}=i h$ is to decide whether or not the process parameter $\theta(t)$ is still on target or has been shifted by the occurrence of a disturbance. By selecting a suitable shift $\theta_{1}$, the decision problem is solved by comparing the two alternatives:

$$
\begin{array}{ll}
H_{0}: & \theta(t)=\theta_{0} \\
H_{1}: & \theta(t)=\theta_{0}+\theta_{1}
\end{array}
$$

Because of the assumptions, it makes sense to use only one fixed decision function

$$
\gamma_{i}=\gamma \in \Gamma \quad \text { with } \quad \gamma:\left\{\left(X_{1}, \ldots, X_{n}\right)\right\} \rightarrow\{0,1\}
$$

in all decision points $t_{i}, i=1,2, \ldots$, where $\Gamma$ denotes the set of admissible decision functions. The error probabilities when using $\gamma$ are denoted by

$$
\begin{aligned}
\alpha & =\mathbf{P}\left(\gamma=1 \mid \theta_{0}\right) \\
\beta & =\mathbf{P}\left(\gamma=0 \mid \theta_{0}+\theta_{1}\right)
\end{aligned}
$$

Now we are in a position to define the monitoring policy investigated here. 
Definition 1 A simple periodic monotoring policy for detecting the occurrence of a random disturbance is given by three quantities $(h, n, \gamma)$, and the following rule: Take every $h \in \mathbb{R}^{+}$hours of operation a random sample of size $n \in \mathbb{N}_{0}$ with $0 \leq n \leq h v$ (where $v$ denotes the number of produced items per hour) from the process output, and decide by means of the decision function $\gamma \in \Gamma$ on the actual state of the system. In case the decision is in favor of a disturbance an alarm is released, the process is inspected and the disturbance is removed in case of a true alarm. Otherwise the process is left alone.

\section{PROCESS PROFITABILITY}

In order to compare different monitoring policies an indicator is needed reflecting the policy's contribution to process profitability. Profitability is defined as the long run profit per item produced which has to be derived taking into account the relevant random interrelations between the single parts of the production process.

Let $G(m)$ be the gain derived from the first $m$ items produced, then

$$
\Pi=\lim _{m \rightarrow \infty} \frac{G(m)}{m}
$$

defines the long run profit per item. Clearly, $\Pi$ is a function of process design, process maintenance, monitoring policy, customer service, and more generally of process management and product marketing. However, aiming at designing only monitoring policies

for detecting random disturbances, the other process features are looked upon as fixed, i.e. $\Pi$ is considered here only as function of the monitoring policy, i.e.

$\Pi=\Pi(h, n, \gamma)$.

The problem is to determine an optimal monitoring policy.

Definition 2 A monitoring policy $\left(h^{*}, n^{*}, \gamma^{*}\right)$ is called optimal with respect to $\Pi$ within the set of admissible monitoring policies $\Gamma=\{(h, n, \gamma)\}$, if

$$
\Pi\left(h^{*}, n^{*}, \gamma^{*}\right) \geq \Pi(h, n, \gamma) \quad \text { for any } \quad(h, n, \gamma) \in \Gamma
$$

In order to find an explicit expression for $\Pi$ the technical and the economic situation has to be described quantitatively.

The economic situation with respect to the output is given by:

$g_{I}=$ expected profit derived from one item produced on target

$g_{I I}=$ expected profit derived from one item produced after occurrence of a disturbance disturbance 
Of course, $g_{I}$ and $g_{I I}$ are complex quantities reflecting a large number of different process and product features including the inherent variations which depend on the state of the process. For instance the variations of the item quality characteristic $X$ are different when producing on target and when producing off target.

The simple monitoring policy includes three actions:

- sampling,

- intervention after false alarm called inspection, and

- intervention after true alarm called renewal.

An economic model describing these actions is given by:

$a^{*} n=$ expected cost for taking and evaluating a sample of size $n$,

$e^{*}=$ expected cost of an inspection after a false alarm, and

$b^{*} \quad=$ expected benefit per renewal after a true alarm.

The three parameters $a^{*}, e^{*}$ and $b^{*}$ are called economic key parameters of a production process.

After a disturbance is detected, the process is renewed. Consecutive renewals divide the process in stochastically equivalent parts which are called renewal cycles. The following random variables refer to one renewal cycle:

$A_{I}=$ number of monitoring actions when operating on target

$A_{F}=$ number of false alarm

$A_{I I}=$ number of monitoring actions after the occurrence of a disturbance

$N=$ number of produced items

$G=$ profit derived from the $N$ produced items

With these quantities, the following expectations are obtained:

$$
\begin{aligned}
& E[G]=g_{I I} E\left[A_{I}+A_{I I}\right] h v+b^{*}-e^{*} E\left[A_{F}\right]-a^{*} n E\left[A_{I}+A_{I I}\right] \\
& E[N]=E\left[A_{I}+A_{I I}\right] h v
\end{aligned}
$$

Hence

$$
\Pi(h, n, \gamma)=\frac{E[G]}{E[N]}=\frac{1}{h v}\left\{\frac{b^{*}-e^{*} E\left[A_{F}\right]}{E\left[A_{I}+A_{I I}\right.}-a^{*} n\right\}+g_{I I}
$$

Any change of a relevant part of the process may be judged beforehand by means of the objective function (15). Moreover, (15) may serve in order to optimize and continuously improve design and methods displayed in the product cycle. 


\section{OPTIMIZATION}

It turns out that from the viewpoint of numerical optimization the objective function(15) has rather ugly properties. One of them is on the one hand the flatness of the objective function in an environment of the optimal solution and on the other hand the steep descent which can be observed when the flat area around the optimal solution is left. The first property implies that an approximate solution is almost as good as an exact solution of the optimization problem. The second property shows that not adopting the integrated and objective-oriented approach may lead to rather inferior solutions.

Besides the numerical problems one has to solve the problem of a user-friendly presentation of optimal monitoring policies. The number of input parameters directly entering the objective function $\Pi$ makes a tabulation of the optimal monitoring policies impos-

sible. However, a tabulation of the economic monitoring policies is an almost necessary condition for competing successfully with the existing "statistical" standards like for instance ISO 8258 [21]. Therefore, one major problem is to find an approximation which is sufficiently accurate and which allows the determination of optimal solutions by means of simple tables and explicit formulas.

\subsection{Approximate Optimal Sampling Interval}

In traditional control charting, there is no way to rationally fixing the sampling interval. This is an major deficiency as it turns out that an inappropriate sampling interval may lead to a sharp decrease of process profitability.

The integrated and objective-oriented approach reflected by (15) enables the derivation of an explicit formula for computing an approximately optimal sampling interval $\hat{h}^{*}$.

For any given sampling plan $(n, \gamma)$, the approximately optimal sampling interval $\hat{h}^{*}(n, \gamma)$ is given in v. Collani [7].

$$
\hat{h}^{*}(n, \gamma)=E[\tau] \frac{\alpha+\frac{a^{*}}{e^{*}} n+\sqrt{\left(\alpha+\frac{a^{*}}{e^{*}} n\right) \frac{b^{*}}{e^{*}}(1-\beta)+\alpha}}{\frac{b^{*}}{e^{*}}+0.5 \alpha-\frac{a^{*}}{e^{*}} n\left(\frac{1}{1-\beta}-0.5\right)}
$$

where $\alpha$ and $\beta$ denote the error probabilities for the sampling plan $(n, \gamma)$.

In industrial environments the approximation (16) is as good as the exact optimal solution because of two reasons:

- The objective function (15) is rather flat in an environment of the optimal solution.

- The optimal sampling interval $h^{*}$ is generally taken in practice only as a guideline. 
The actually used sampling interval is rounded and adjusted to the needs of the respective situation.

Formula (16) may be taken to complete in an appropriate way any control chart, e.g. a control chart taken from ISO 8258 and given by the sampling plan $(n, \gamma)$. However, the objective function (15) also enables the determination of an optimal sampling plan. In v. Collani [7] a methodology is developed for determining approximately optimal sampling plans $\left(\hat{n}^{*}, \hat{\gamma}^{*}\right)$ for various situations with respect to the underlying distribution model.

\subsection{Approximately Optimal Sampling Plan}

In v. Collani [7] it is shown under rather general conditions that the optimal sampling plan $\left(n^{*}, k^{*}\right)$ may be approximately determined by minimizing a loss function $L(n, k)$, which is obtained as a limit of the optimum of (15) when $\lambda h$ tends to 0 . Actually, $L(n, k)$ represents the losses due to not detecting immediately a disturbance (first factor) and due to sampling and to false alarms (second factor).

$$
L(n, k)=\left(\frac{1}{1-\beta(n, k)}-\frac{1}{2}\right) \cdot(a n+\alpha(n, k))
$$

where $a=\frac{a^{*}}{e^{*}}$.

By means of $L(n, k)$ the above mentioned major problem is solved at least with respect to the sampling plan $(n, k)$ :

- There is only one economic parameter and, depending on the model, only few distributional parameters explicitly entering $L(n, k)$, thus allowing a comprehensive tabulation of approximately optimal sampling plans for many situations.

For deriving approximately optimal sampling plans two of the three economic key parameters must be necessarily known. These are:

sampling cost $\quad a^{*}$

inspection cost $e^{*}$

If sampling is expensive compared with inspections yielding a large value of the parameter $a$ in (17), then the optimal sample size is small since a larger false alarm rate is tolerable from an economic point of view. On the other hand, if sampling is cheap compared with an inspection yielding a small value of $a$, the optimal sample size gets larger and consequently the false alarm rate smaller.

\subsection{Normal, one-sided case, fixed variance}

The relevant parts of the process are the quality characteristic $X$, its distribution, the 
target value and the effect of a disturbance. The one-sided normal case with fixed variance is selected, if the quality characteristic $X$ is a measurable variable with a one-sided specification where the disturbance affects only the process mean given by the expectation $E[X]$ but not the process variability given by a constant variance $V[X]$, which can be assumed as being known.

$\begin{array}{ll}\text { quality characteristic } & X_{t}\left(\mu(t), \sigma^{2}\right) \\ \text { distribution } & N\left(\mu(t), \sigma^{2}\right) \\ \text { target value } & \mu_{0} \\ \text { effect of disturbances } & P\left(\mu(t)=\mu_{0}+\delta \sigma\right)=1\end{array}$

The following process input parameters have to be specified by the user:

$\begin{array}{ll}\text { target value } & \mu_{0} \\ \text { variance } & \sigma^{2} \\ \text { shift parameter } & \delta\end{array}$

The decision function is completely determined by the so-called control limit $c$ and given by the following well-known expression where $\left(X_{1}, \ldots, X_{n}\right)$ denotes the sample of size $n$ and $\bar{X}$ the arithmetic mean of the sample.

$$
\gamma\left(X_{1}, \cdots, X_{n}\right)=\left\{\begin{array}{lll}
0 & \text { for } & \frac{\bar{X}-\mu_{0}}{\sigma} \sqrt{n}<c \\
1 & \text { for } & \frac{\bar{X}-\mu_{0}}{\sigma} \sqrt{n} \geq c
\end{array}\right.
$$

The approximate optimal sampling plan $\left(\hat{n}^{*}, \hat{c}^{*}\right)$ is obtained in the following simple way.

- Calculate $a_{0}=\frac{a^{*}}{e^{*}} \cdot \frac{1}{\delta^{2}}$ and take for given $a_{0}$ the quantities $y$ and $z$ from Table 1 and set

$$
\begin{aligned}
& \hat{n}^{*}=\text { closest positive integer to }\left(\frac{y}{\delta}\right)^{2} \\
& \hat{c}^{*}=z
\end{aligned}
$$

Below a detail of Table 1 is displayed.

\begin{tabular}{|c||ccccc|}
\hline$a_{0}$ & 0.0001 & 0.0005 & 0.0010 & 0.0050 & 0.01 \\
\hline \hline$y$ & 4.70283 & 4.16945 & 3.91402 & 3.23682 & 2.89647 \\
\hline$z$ & 3.47853 & 3.02028 & 2.80396 & 2.214127 & 1.96448 \\
\hline
\end{tabular}

Table 1: Normal, one-sided case, fixed variance

\subsection{Normal, two-sided symmetric case, fixed variance}

This case (see v. Collani [5], [6]) is assumed when the quality characteristic is measurable with two specifications and when a disturbance affects only the process mean $E[X]$ but not the process variance $V[X]$ which consequently is assumed to be known. Moreover, negative and positive shifts are equally probable. 
quality characteristics $\quad X_{t}\left(\mu(t), \sigma^{2}\right)$

distribution $\quad N\left(\mu(t), \sigma^{2}\right)$

target value $\quad \mu_{0}$

effects of disturbances $\quad P\left(\mu(t)=\mu_{0}-\delta \sigma\right)=0.5$

$P\left(\mu(t)=\mu_{0}+\delta \sigma\right)=0.5$

In this case the same set of process input parameters have to be specified as in the preceding case.

target value

variance

$\mu_{0}$

shift parameter $\delta$

The decision function is given by:

$$
\gamma\left(X_{1}, \cdots, X_{n}\right)=\left\{\begin{array}{ll}
0 & \text { for } \\
1 & \text { for }
\end{array} \mid \begin{array}{l}
\frac{\bar{X}-\mu_{0}}{\sigma} \sqrt{n} \\
\frac{\bar{X}-\mu_{0}}{\sigma} \sqrt{n} \mid \geq c
\end{array}\right.
$$

The approximate optimal sampling plan $\left(\hat{n}^{*}, \hat{c}^{*}\right)$ can be determined as follows.

- Calculate $a_{0}=\frac{a^{*}}{e^{*}} \cdot \frac{1}{\delta^{2}}$ and take for given $a_{0}$ the quantities $y$ and $z$ from Table 2 and set:

$$
\begin{aligned}
& \hat{n}^{*}=\text { closest positive integer to }\left(\frac{y}{\delta}\right)^{2} \\
& \hat{c}^{*}=z
\end{aligned}
$$

where a detail of Table 2 is given below.

\begin{tabular}{|c||ccccc|}
\hline$a_{0}$ & 0.0001 & 0.0005 & 0.0010 & 0.0050 & 0.01 \\
\hline \hline$y$ & 4.91206 & 4.40830 & 4.16945 & 3.54520 & 3.23682 \\
\hline$z$ & 3.66052 & 3.22443 & 3.02027 & 2.49551 & 2.24127 \\
\hline
\end{tabular}

Table 2: Normal, two-sided symmetric case, fixed variance

\subsection{Normal, two-sided asymmetric case, fixed variance}

The process characteristics are the same as in the preceding case except for the shifts and frequencies of negative and positive shifts after occurrence of a disturbance which are now assumed to be not equal.

$$
\begin{array}{ll}
\text { quality characteristics } & X_{t}\left(\mu(t), \sigma^{2}\right) \\
\text { distribution } & N\left(\mu(t), \sigma^{2}\right) \\
\text { target value } & \mu_{0} \\
\text { effects of disturbances } & P\left(\mu(t)=\mu_{0}-\delta_{1} \sigma\right)=w \\
& P\left(\mu(t)=\mu_{0}+\delta_{2} \sigma\right)=1-w
\end{array}
$$

The corresponding set of process input parameters includes the shift parameters and the probability $w$ of a negative shift. 
target value

variance

$\mu_{0}$

negative shift parameter

positive shift parameter $\quad \delta_{2}$

negative shift probability $w$

In this case the decision function takes into account the asymmetry of the shifts by admitting two different control limits $c_{1}$ and $c_{2}$.

$$
\gamma\left(X_{1}, \cdots, X_{n}\right)=\left\{\begin{array}{lll}
0 & \text { for } & -c_{1}<\frac{\bar{X}-\mu_{0}}{\sigma} \sqrt{n}<c_{2} \\
1 & \text { for } \quad \frac{\bar{X}-\mu_{0}}{\sigma} \sqrt{n} \leq c_{1} \text { or } \frac{\bar{X}-\mu_{0}}{\sigma} \sqrt{n} \geq c_{2}
\end{array}\right.
$$

The approximate optimal sampling plan $\left(\hat{n}^{*}, \hat{c}^{*}\right)$ is determined in a similar way as in the preceding cases:

- Calculate $a_{0}=\frac{a^{*}}{e^{*}} \cdot \frac{1}{\delta^{2}}$ and $\Delta=\frac{\delta_{1}}{\delta_{2}}$ and take for given $a_{0}, \Delta$ and $w$ the quantities $y$, $z_{1}$ and $z_{2}$ from Table 3 and set

$$
\begin{aligned}
& \hat{n}^{*}=\text { closest positive integer to }\left(\frac{y}{\delta}\right)^{2} \\
& \hat{c}_{1}^{*}=z_{1} \\
& \hat{c}_{2}^{*}=z_{2}
\end{aligned}
$$

where a detail of Table 3 is given below:

\begin{tabular}{|c||ccccc|}
\hline \multicolumn{5}{|c|}{$\Delta=0.25$} & \multicolumn{3}{c|}{$w=0.2$} \\
\hline$a_{0}$ & 0.0001 & 0.0005 & 0.0010 & 0.0050 & 0.01 \\
\hline \hline$y$ & 9.97406 & 7.22355 & 6.10928 & 4.25170 & 3.62780 \\
\hline$z_{1}$ & 2.79716 & 2.29700 & 2.07814 & 1.57406 & 1.35386 \\
$z_{2}$ & 5.42927 & 4.09091 & 3.55880 & 2.59313 & 2.21450 \\
\hline
\end{tabular}

Table 3: Normal, two-sided asymmetric case, fixed variance

\subsection{Normal, two-sided symmetric case}

This case is similar equal to the second case except for the fact that a disturbance affects not only the process mean $E[X]$ but also the process variability $V[X]$. For details see v. Collani[8] and v. Collani \& Treml [11]. The process characteristics are the following.

$\begin{array}{ll}\text { quality characteristics } & X_{t}\left(\mu(t), \sigma^{2}(t)\right. \\ \text { distribution } & N\left(\mu(t), \sigma^{2}(t)\right) \\ \text { target value for process mean } & \mu_{0} \\ \text { target value for process variability } & \sigma_{0}^{2} \\ \text { effects of disturbances } & P\left(\mu(t)=\mu_{0}-\delta_{1} \sigma_{0}\right)=0.5 \\ & P\left(\mu(t)=\mu_{0}+\delta_{1} \sigma_{0}\right)=0.5 \\ & P\left(\sigma^{2}(t)=\left(\delta_{2} \sigma_{0}\right)^{2}\right)=1.0\end{array}$

The set of process input parameters to be specified by the user is given below. 
target value for process mean

target value for process variability

shift parameter for process mean

shift parameter for process variance

$\mu_{0}$
$\sigma_{0}^{2}$
$\delta_{1} \geq 0$
$\delta_{2}>1$

Instead of using a $\left(\bar{X}, S^{2}\right)$-decision rule, as proposed e.g. by the ISO standard [21], the following simple decision function is recommended.

$$
\gamma\left(X_{1}, \cdots, X_{n}\right)=\left\{\begin{array}{lll}
0 & \text { for } & \frac{1}{n} \sum_{i=1}^{n}\left(\frac{X_{i}-\mu_{0}}{\sigma_{0}}\right)^{2}<c \\
1 & \text { for } & \frac{1}{n} \sum_{i=1}^{n}\left(\frac{X_{i}-\mu_{0}}{\sigma_{0}}\right)^{2} \geq c
\end{array}\right.
$$

For determining an approximately optimal sampling $\left(\hat{n}^{*}, \hat{c}^{*}\right)$ the following algorithm can be used.

- Calculate $a=\frac{a^{*}}{e^{*}}$ and take for given $a, \delta_{1}$ and $\delta_{2}$ the approximately optimal sampling plan $\hat{n}^{*}$ and $\hat{c}^{*}$ from Table 4 .

Below there is a detail of Table 4 .

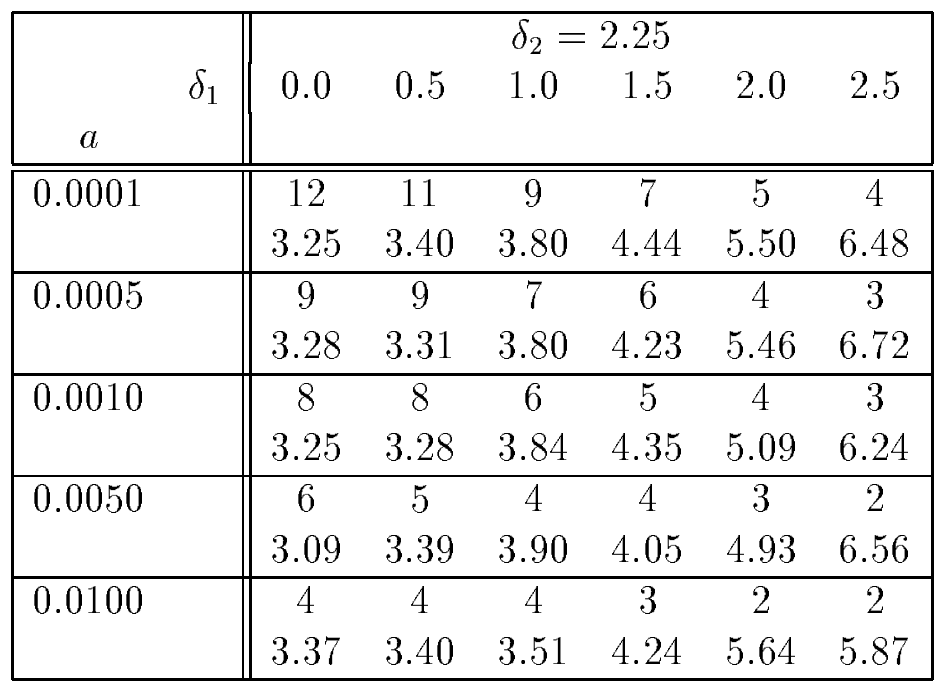

Table 4: Normal, two-sided and symmetric case

\subsection{Poisson case}

If item quality is determined by the number of nonconformities then the Poisson model is often appropriate. For details compare v. Collani \& Dräger [13] In this case the following process characteristics are obtained.

$$
\begin{array}{ll}
\text { quality characteristics } & X_{t}(\mu(t)) \\
\text { distribution } & P o(\mu(t)) \\
\text { target value } & \mu_{0} \\
\text { effect of disturbances } & \left.P\left(\mu(t)=\delta \mu_{0}\right)\right)=1.0
\end{array}
$$


The set of process input parameters to be specified by the user is as follows.

target value $\quad \mu_{0}$

shift parameter $\delta>1$

In this case the decision function is based on the total number of nonconformities in the sample:

$$
\gamma\left(X_{1}, \cdots, X_{n}\right)=\left\{\begin{array}{lll}
0 & \text { for } & \sum_{i=1}^{n} X_{i}<c \\
1 & \text { for } & \sum_{i=1}^{n} X_{i} \geq c
\end{array}\right.
$$

The approximately optimal sampling plan $\left(\hat{n}^{*}, \hat{c}^{*}\right)$ is obtained by means of Table 5 .

- Calculate $a=\frac{a^{*}}{e^{*}} \cdot \frac{1}{\mu_{0}}$ and $\Delta=\frac{\mu_{1}}{\mu_{0}}$ and take for given $a$ and $\Delta$ the quantities $y$ and $z$ from Table 5 . Then set

$$
\begin{aligned}
& \hat{n}^{*}=\text { closest positive integer to } \frac{y}{\mu_{0}} \\
& \hat{c}^{*}=z
\end{aligned}
$$

\begin{tabular}{|c|c|c|c|c|c|}
\hline $\begin{array}{ll} & \Delta \\
a_{0} & \\
\end{array}$ & 1.50 & 2.00 & 3.00 & 4.00 & 5.00 \\
\hline 0.0005 & $\begin{array}{c}59.237 \\
79\end{array}$ & $\begin{array}{c}21.646 \\
36\end{array}$ & $\begin{array}{c}7.832 \\
18\end{array}$ & $\begin{array}{c}4.493 \\
13\end{array}$ & $\begin{array}{c}2.832 \\
10\end{array}$ \\
\hline 0.0010 & $\begin{array}{c}49.792 \\
66\end{array}$ & $\begin{array}{c}18.757 \\
31\end{array}$ & $\begin{array}{c}7.031 \\
16\end{array}$ & $\begin{array}{c}4.223 \\
12\end{array}$ & $\begin{array}{c}2.580 \\
9\end{array}$ \\
\hline 0.0050 & $\begin{array}{c}26.953 \\
35\end{array}$ & $\begin{array}{c}12.402 \\
20\end{array}$ & $\begin{array}{c}4.954 \\
11\end{array}$ & $\begin{array}{c}2.848 \\
8\end{array}$ & $\begin{array}{c}2.107 \\
7\end{array}$ \\
\hline 0.0100 & $\begin{array}{c}18.057 \\
23\end{array}$ & $\begin{array}{c}9.433 \\
15\end{array}$ & $\begin{array}{c}4.127 \\
9\end{array}$ & $\begin{array}{c}2.566 \\
7\end{array}$ & $\begin{array}{c}1.846 \\
6\end{array}$ \\
\hline 0.0500 & $\begin{array}{c}0.000 \\
0\end{array}$ & $\begin{array}{c}3.455 \\
5\end{array}$ & $\begin{array}{c}2.497 \\
5\end{array}$ & $\begin{array}{c}1.580 \\
4\end{array}$ & $\begin{array}{c}0.964 \\
3\end{array}$ \\
\hline
\end{tabular}

where a detail of Table 5 is given below.

Table 5: Poisson case

\subsection{Binomial case}

If each item can be classified either as conforming or nonconforming then the binomial model is used for the item quality characteristic. This case is investigated in detail in v. Collani \& Dräger [12]. The process characteristics are the following.

$$
\begin{array}{ll}
\text { quality characteristic } & X_{t}(p(t)) \\
\text { distribution } & B i(1, p(t)) \\
\text { target value } & p_{0} \\
\text { effect of disturbances } & P\left(p(t)=p_{1}\right)=1.0
\end{array}
$$

The set of process input parameters to be specified is given below. 
target value $\quad p_{0}$

shifted value $\quad p_{1}>p_{0}$

Similar as in the Poisson case the number of nonconforming items in the sample is used for making a decision.

$$
\gamma\left(X_{1}, \cdots, X_{n}\right)=\left\{\begin{array}{lll}
0 & \text { for } & \sum_{i=1}^{n} X_{i}<c \\
1 & \text { for } & \sum_{i=1}^{n} X_{i} \geq c
\end{array}\right.
$$

The approximately optimal sampling plan $\left(\hat{n}^{*}, \hat{c}^{*}\right)$ is determined in the following way.

- Calculate $a=\frac{a^{*}}{e^{*}}$ and $\Delta=p_{1}-p_{0}$ and take for given $a, \Delta$ and $p_{0}$ the approximately optimal sampling plan $\left(\hat{n}^{*}, \hat{c}^{*}\right)$ from Table 6.

where a detail of Table 6 is displayed below.

\begin{tabular}{|c|c|c|c|c|c|}
\hline & \multicolumn{5}{|c|}{$\Delta=0.05$} \\
\hline$p_{0}$ & 0.01 & 0.02 & 0.04 & 0.06 & 0.08 \\
\hline 0.0005 & $(85,3)$ & $(127,6)$ & $(158,11)$ & $(201,18)$ & $(227,25)$ \\
\hline 0.0010 & $(59,2)$ & $(87,4)$ & $(119,8)$ & $(149,13)$ & $(157,17)$ \\
\hline 0.0050 & $(5,0)$ & $(29,1)$ & $(37,2)$ & $(42,3)$ & $(35,3)$ \\
\hline 0.0100 & $(4,0)$ & $(8,0)$ & $(11,0)$ & $(0,0)$ & $(0,0)$ \\
\hline
\end{tabular}

Table 6: Binomial case

\section{CONCLUSION}

A quantitative model of the relevant technical and economical features of a process and their relations constitutes a necessary condition for designing, maintaining and continuously improving a manufacturing process and predicting its profitability. Such a model taking into account the entirety of features including the inherent uncertainty enables

- to judge any relevant process part with respect to its contribution to profitability,

- to react faster and in an appropriate way to changes with respect to relevant parts,

- to evaluate new technological developments with respect to their usefulness.

There is, however, also a problem which should not be concealed. The above outlined approach for improving process profitability assumes sufficiently educated experts in stochastic modeling. Scientists who are able to investigate, analyze and model the laws or the structure of uncertainty. At many of our universities mathematicians are educated in probability theory and stochastic processes and often it is assumed that either of the fields 
are part of mathematics. Besides, there are statisticians who are taught how to select, apply and to some extent also to develop statistical methods for estimating and testing purposes, where statistics is considered as a mere methodology for handling large data sets. Unfortunately, neither mathematics (which is used in stochastics as the appropriate language) nor a statistical methodology are sufficient to solve the problems associated with the aim of modeling profitability for a purposeful planning of future developments.

The late W. Edward Deming notes in his masterpiece "Out of the Crisis", p. 132:

"The teaching of pure statistical theory in universities, including the theory of probability and related subjects, is almost everywhere excellent. Application to enumerative studies is mostly correct, but application to analytic problems - planning for improvement of tomorrow's run, next years crop - is unfortunately, however, in many textbooks deceptive and misleading."

Besides mathematics as language, computers are indispensable tools for solving the related computational problems. However, for solving the actual problems not computers, but experts in modeling uncertainty are necessary. In his book Deming (p.133) throws some light on the relation between statistics (or as I prefer to call it stochastics) and the computer:

"People with master's degrees in statistical theory accept jobs in industry and government to work with computers. It is a vicious cycle. Statisticians do not know what statistical work is, and are satisfied to work with computers. People that hire statisticians likewise have no knowledge about statistical work, and somehow suppose that computers are the answer. Statisticians and management thus misguide each other and keep the vicious cycle rolling."

What is needed for any manufacturing process is a model of profitability from the very beginning of its operation as main declaration of the relevant performance characteristics. The model should use up any available information and should be continuously improved with any additional knowledge obtained during process operation. The modeling process should be based on a purposeful data recording and data processing. It enables rational decisions on any technical and managerial question related to the process in question. 


\section{References}

[1] R.E.BARLOW and F.PROSCHAN, Statistische Theorie der Zuverlässigkeit. Harri Deutsch, Frankfurt/Main (1978).

[2] F. BEICHELT, Zuverlässigkeit und Instandhaltungstheorie. Teubner Verlag, Stuttgart (1993).

[3] F. BEICHELT, Optimum Replacement Policies Under Stochastics Repair Cost Development. Economic Quality Control 12, 173-181 (1997).

[4] G.E.P.BOX AND A.LUCEÑO, Statistical Control by Monitoring and Feedback Adjustment. Wiley, New York (1997).

[5] E.v. COLLANI, The Economic Design of Control Charts. Teubner, Stuttgart (1989).

[6] E.v. COLLAni, A Simple Procedure to Determine the Economic Design of an $\bar{X}$-Control Chart. Journal of Quality Technology, 18/3, 145-151 (1986).

[7] E.v. COLLANI, Determination of the Economic Design of Control Charts Simplified. In: Eds. K.S.Al-Sultan \& M.A. Rahim: Optimization in Quality Control, Kluwer, Boston (1997).

[8] E.v. COLLANI, Control of Production Processes Subject to Random shocks. Annals of Operation Research 91, 289-404 (1999).

[9] E.v. COLLANI and Ch. WEIGAND, Economic Machine Adjustment in the Case of Product Screening. Statistical Papers 33, 171-184 (1992).

[10] E.v. COLLANI, E.M.SANIGA and Ch.WEIGAND, Economic Adjustment Designs for $\bar{x}$ Control Charts. IIE Transactions, 26, 37-43 (1994).

[11] E.v. COLLANI and J. TREML, Control of a Two-Dimensional Process-QualityIndicator by means of a Screening Procedure. Economic Quality Control 8, 167-194 (1993).

[12] E.v.COLLANI and K.DRÄGER, A Simplified Economic Design of Control Charts for Monitoring the Nonconforming Probability. Economic Quality Control 10, 231-276 (1995).

[13] E.v. COLLANI and K.DRÄGER, The Economic Design of Control Charts for Monitoring the Number of Nonconformities. Economic Quality Control 12, 15-46 (1997). 
[14] E.v. COLLANI and V.DRAGALIN, A Simplified Economic Design of CUSUM Charts for Monitoring a Normally Distributed Process Mean. Allgemeines Statistisches Archiv (ASTA) 84, 217-293 (2000).

[15] R.J.M.M. DOES, K.C.B. ROES and A.TRIP, Statistical Process Control in Industry. Kluwer Academic Publishers, Dordrecht (1999).

[16] M. DUMiTRESCU, On the Robustness of MCEWMA Control Charts for AR(1) Processes. Economic Quality Control 10, 223-230 (1995).

[17] M. DUMITRESCU, Mathematical Basis of Process Monitoring. ASU Technical Paper Series 98-6, Quality and Reliability Engineering Group, Arizona State University (1998).

[18] A.J. DUNCAN, The Economic Design of $\bar{X}$ Control Charts Used to Maintain Current Control of Process. Journal of the American Statistical Association 51, 228-242 (1956).

[19] P.FRAHM, Combining Economic Design of $\bar{X}$-Charts and Block Replacement Under Weibull Shock Models. Economic Quality Control, 10/2, 77-115 (1995).

[20] C. HO and K.E. CASE, Economic Design of Control Charts: A Literature Review for 1981-1991. Journal of Quality Technology, 26/1, 39-53 (1994).

[21] International Standard ISO 8258:1991 (E), Shewhart Control Charts. Genève, Switzerland (1991).

[22] G. KIESMÜLLER, Controlling a Process with Three Different States. Advances in Stochastic Models for Reliability, Quality and Saftey. Birkhäuser Verlag, Boston (1998).

[23] G. KIESMÜLlER, Most Profitable Process Control - An Integrated Approach. Economic Quality Control 14, 5-18 (1999).

[24] G. KIESMÜLLER, Most Profitable Inspection Policies. Economic Quality Control 14, 65-83 (1999).

[25] G. KIESMÜLLER, Most Profitable Sampling Policies. Economic Quality Control 14, 105-122 (1999).

[26] T.J.LORENZEN and L.C. VANCE, Th Economic Design of Control Charts: A Unified Approach. Technometrics 28, 3-10 (1986).

[27] D.C.MONTGOMERY, The Economic Design of Control Charts: A Review and Literature Survey. Journal of Quality Technology, 12, 75-87 (1980). 
[28] M.A. RAHIM, Economic Design of $\bar{X}$ Control Charts Assuming Weibull In-Control Times. Journal of Quality Technology, 225, 296-305 (1993).

[29] M.A. RAHIM and P.K. BANERJEE, A Generalized Model for the Economic Design of $\bar{x}$-Control Charts for Production systems with increasing Failure Rate and Early Replacement. Naval research Logistics, 40, 787-809 (1993). 
